\title{
Acetylcholine Mediates AMPK-Dependent Autophagic Cytoprotection in H9c2 Cells During Hypoxia/Reoxygenation Injury
}

\author{
Mei Zhao Lei Sun $^{\mathrm{a}}$ Xiao-Jiang Yu Yi Miao $^{\mathrm{a}}$ Jin-Jun Liu ${ }^{\mathrm{a}}$ Hao Wang ${ }^{\mathrm{a}}$ Jun Ren ${ }^{\mathrm{b}}$ \\ Wei-Jin Zanga
}

${ }^{a}$ Department of Pharmacology, College of Medicine, Xi'an Jiaotong University, Xi'an, Shaanxi; ${ }^{b}$ Center for Cardiovascular Research and Alternative Medicine, College of Health Sciences, University of Wyoming, Laramie, Wyoming

\section{Key Words}

Acetylcholine • Autophagy • Hypoxia/reoxygenation • Muscarinic receptor • AMPK

\begin{abstract}
Background: Acetylcholine (ACh), a neurotransmitter of vagal nerve, offers tolerance to ischemia/reperfusion injury. Given the regulation of autophagy in cardioprotection, this study was to examine the role of autophagy in ACh-elicited protection against hypoxia/ reoxygenation (HR) injury. Methods: H9c2 cells were subjected to HR injury. Autophagy was determined by transmission electron microscopy, MDC staining and western blot. MTT kit, LDH and CK release, ATP content and TUNEL assay were used to evaluate cardiomyocytes injury. Atg7 and AMPK knockdown was performed with siRNA transfection. Results: Following 4, 8, 12 and $16 \mathrm{~h}$ reoxygenation, autophagosomes were decreased along with reduced cell viability. ACh during $4 \mathrm{~h}$ reoxygenation facilitated autophagy as evidence by increased autophagosomes and MDC labeling autophagic vacuoles. H9c2 cells treated with ACh also underwent a biochemical changes by increased ratio of LC3-II/LC3-I and autophagy flux (decreased p62), while muscarinic receptor antagonist atropine suppressed these effects. Induction of autophagy was correlated with enhanced cell survival and decreased apoptosis. Autophagy inhibition with chloroquine and Atg7 siRNA significantly attenuated ACh-induced cytoprotection. ACh-elicited autophagy activation could be related to increased AMPK phosphorylation and decreased mTOR phosphorylation. AMPK siRNA exhibited an elevation in mTOR phosphorylation and reduced the ratio of LC3-II/LC3-I. Importantly, AMPK knockdown desensitized H9c2 cells to ACh-mediated protection. Conclusions: These data provided first evidence that ACh-induced autophagy elicited cytoprotective effects through muscarinic receptor activated-AMPK-mTOR pathway, and suggested a novel mechanism of ACh-induced tolerance against HR injury.
\end{abstract}

Copyright $@ 2013$ S. Karger AG, Basel 


\section{Introduction}

Ischemic heart disease is a leading cause of death worldwide. However, the restoration of blood flow exacerbates the insult sustained from ischemia (termed "ischemia/ reperfusion injury") [1]. It is conceived that reperfusion injury per se contributes to almost one-fourth of mortality in patients with myocardial infarction [2]. Notably, apoptosis and necrosis are mainstays contributable to ischemia/reperfusion injury. However, autophagy, as another alternative mechanism, has caused an increasing awareness to determine cell fate [3]. Autophagy is an intracellular evolutionarily conserved catabolic process to degrade and recycle long-lived proteins and damaged organelles [4]. Autophagy activity remains at a low level under physiological condition and can be activated in response to stress condition including starvation and oxidative stress. Excessive auotphagy, nevertheless, leads to programmed cell death $[5,6]$. As a "housekeeper", autophagy plays a unique role in maintaining cell homeostasis including ATP and protein synthesis [7]. Recent evidence has indicated an elevated autophagy during ischemia, while suppression of autophagy is accompanied with aggravated injury $[8,9]$. A number of autophagic induction models such as Atg7 overexpression have shown to improve myocardial function [10]. Cardioprotective agents such as adenosine and resveratrol have also been found to reduce damage dependent on autophagy induction $[11,12]$. These results suggest that autophagy may play a role in switching cell death signals into survival signals.

It is now widely accepted that imbalanced autonomic nervous system especially reduced vagal activity is an independent risk factor of cardiovascular diseases including heart failure, hypertension and myocardial infarction $[13,14]$. Vagal nerve stimulation has been implicated in chronic heart failure patients [15]. Likewise, the vagal neurotransmitter acetylcholine $(\mathrm{ACh})$ prevents hypoxia-induced injury in various cell types such as embryonic stem cells and HEK293 cells $[16,17]$. Endogenous ACh release was inhibited with ischemia [18]. In contrast, ACh from vagal nerve stimulation suppressed left ventricular remodeling, thus reliefs the progression of myocardial infarction [19]. Along the same line, our previous study has demonstrated that exogenous ACh improves contractile function and cell viability, consolidating the cardiovascular therapeutic benefit of ACh [20]. The suppression of apoptosis and ROS generation has been shown to involved in ACh-induced ischemic tolerance $[21,22]$. However, the underlying cardioprotective mechanism of ACh is not fully defined. In addition, more research is necessary for extensive clinically application of vagal therapeutics.

As ACh generates a survival signal, little information is available about the relationship between ACh and autophagy. Therefore, we employed H9c2 cells to identify the involvement of autophagy with ACh during cardiac dysfunction. We went on to examine the possible mechanism(s) of action in ACh-facilitated autophagy with a focus on AMPK-mTOR pathway.

\section{Materials and Methods}

\section{Materials}

DMEM and FBS were purchased from Thermo Fisher. ACh, atropine, chloroquine, penicillin, streptomycin, MTT, DMSO, monodansylcadaverine (MDC) and 4', 6-Diamidino-2-phenylindole (DAPI) were obtained from Sigma-Aldrich. ACh, atropine and chloroquine were dissolved in phosphate buffer saline. Drug concentrations are expressed as the final molar concentration within the culture dish. Anti-GAPDH, anti-LC3, anti-p62 were purchased from Sigma-Aldrich. Anti-Atg7, anti-AMPK, anti-p-AMPK, anti-mTOR, anti-p-mTOR and horseradish peroxidase-linked secondary antibodies were from Cell Signaling Technology.

\section{Cell culture}

H9c2 cells, derived from rat embryonic ventricular cardiomyocytes, were cultured in DMEM contained with $10 \%$ (v/v) FBS, $100 \mathrm{U} / \mathrm{ml}$ penicillin and $100 \mu \mathrm{g} / \mathrm{ml}$ streptomycin at $37^{\circ} \mathrm{C}$ in an atmosphere of $95 \%$ air and $5 \% \mathrm{CO}_{2}$. Cells were subjected to experimental procedures at $80 \%$ confluency. 
Fig. 1. Schematic presentation of the experimental procedures. (A) H9c2 cells were starved with DMEM for $12 \mathrm{~h}$ when cells reached about $80 \%$ confluency. Then cells were subjected to $8 \mathrm{~h}$ hypoxia followed by $4 \mathrm{~h}$ reoxygenation. ACh $\left(10^{-6} \sim 10^{-3} \mathrm{M} / 10^{-6} \mathrm{M}\right)$ with or without other inhibitors including atropine $\left(10^{-4}\right.$ M) and chloroquine $(50 \mu \mathrm{M})$ were administered during $4 \mathrm{~h}$ reoxygenation. Con: control; HR: hypoxia/reoxygenation; HR+ACh: acetylcholine with HR; HR+ACh+Atro: acetylcholine + atropine with HR; $\mathrm{HR}+\mathrm{ACh}+\mathrm{Cq}$ : acetylcholine + chloroquine with HR. (B) Protocol for siRNA transfection. Cells were transfected with siRNA for $48 \mathrm{~h}$ when reaching about $60 \%$ confluency and stabilized with DMEM for $12 \mathrm{~h}$. Then cells were subjected to HR and treated with ACh during reoxygenation.

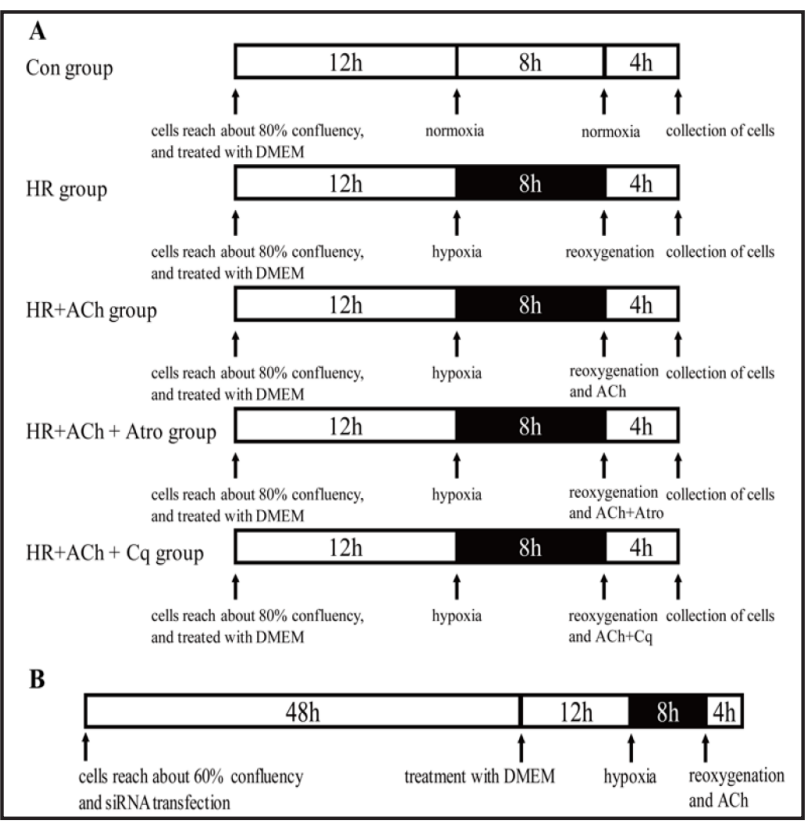

Hypoxia/reoxygenation (HR) and drug administration

H9c2 cells were starved in serum-free DMEM for $12 \mathrm{~h}$ and then incubated in a hypoxic incubator containing $94 \% \mathrm{~N}_{2}, 5 \% \mathrm{CO}_{2}$ and $1 \% \mathrm{O}_{2}$ (monitored by an oxygen probe) with a modified ischemia-mimetic solution (in mM: $\mathrm{NaCl}, 135 ; \mathrm{KCl}, 8 ; \mathrm{MgCl}_{2}, 0.5 ; \mathrm{NaH}_{2} \mathrm{PO}_{4}, 0.33$; HEPES, 5.0; $\mathrm{CaCl}_{2}, 1.8 ; \mathrm{Na}^{+}$-lactate, 20; $\mathrm{pH}$ 6.8) to simulate ischemia. After $8 \mathrm{~h}$ hypoxia, cells were rapidly transferred into a normoxic incubator with DMEM for reoxygenation $(4,8,12,16 \mathrm{~h}$ or $4 \mathrm{~h})$. Cells were treated with ACh $\left(10^{-6}-10^{-4} \mathrm{M} / 10^{-6} \mathrm{M}\right)$ with or without other inhibitors including atropine $\left(10^{-4} \mathrm{M}\right)$ and chloroquine $(50 \mu \mathrm{M})$ during $4 \mathrm{~h}$ reoxygenation. As shown in Fig. 1A, this study included five experimental groups. After the treatment, cells were harvested and analyzed.

\section{siRNA transfection}

H9c2 cells were transfected with siRNA targeting Atg7, AMPK and control siRNAs (GenePharma) with TurboFect siRNA transfection reagent (Thermo Scientific) following the manufacturer's instructions. After $48 \mathrm{~h}$, cells were harvested and analyzed by western blot to confirm the knockdown efficiency of Atg7 and AMPK. Parallel cells were subjected to HR and treated with ACh during reoxygenation as shown in Fig. 1B.

\section{Cell survival assay}

After HR treatment, MTT was added to incubate for $4 \mathrm{~h}$ at $37^{\circ} \mathrm{C}$ and solubilized with $150 \mu \mathrm{l}$ DMSO. The absorbance was read at $492 \mathrm{~nm}$ using a multi-well scanning spectrophotometer reader. Cells in the control group were considered $100 \%$ viable.

\section{Measurement of ATP content}

After the experiment, ATP bioluminescent assay kit (Beyotime Biotech) was used to measure intracellular ATP content according to the manufacturer's instructions. The results were expressed relative to the control (X-fold) in the same experiment.

\section{Measurement of $L D H$ and $C K$}

Briefly, after the treatments, the supernatant was collected to measure the LDH and CK levels with biochemical detecting system (Olympus, AU2700). The results were expressed relative to the control (X-fold) in the same experiment.

\section{TUNEL assay}

The apoptotic cells were detected by TUNEL system according to the manufacturer's instructions (Promega). DAPI was used to stain nuclei. Samples were analyzed using a fluorescence microscopy (TE2000, 
Fig. 2. Cell viability and autophagosome formation following HR. (A) Cell viability was measured using MTT assay. (B) Quantitative analysis of autophagosome number by TEM. Ten fields for each sample were randomly examined. Duration of reoxygenation: hypoxia $8 \mathrm{~h}$ and reoxygenation 4, 8, 12 and 16 h. (C) Photomicrographs exhibiting representative samples from control, HR 4 and 16 $\mathrm{h}$ groups. Arrows denoted autophagosomes. Scale bar: 0.25 $\mu \mathrm{m} .{ }^{* *} \mathrm{P}<0.01$ vs. control group. $\mathrm{n}=6$ per group.

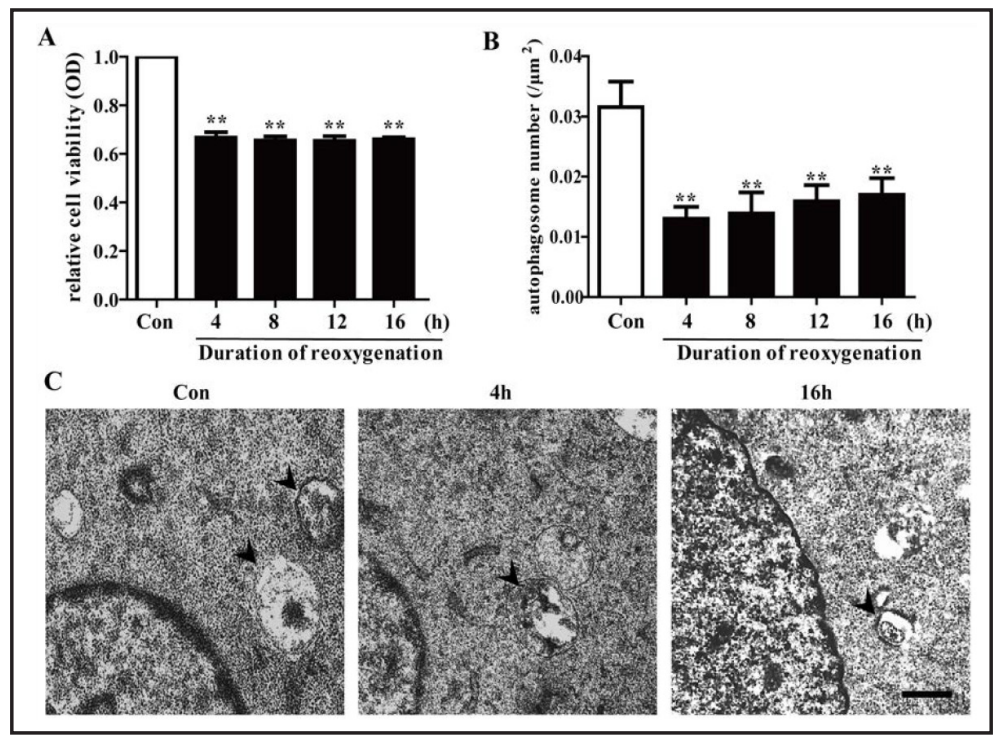

Nikon). Apoptotic cells were counted using the NIH Image 1.63 software package (National Institutes of Health, Bethesda, MD, USA).

Transmission electron microscopy (TEM)

Harvested cells were fixed with $2.5 \%$ glutaraldehyde along with $4 \%$ paraformaldehyde in $0.1 \mathrm{M}$ phosphate buffer for $2 \mathrm{~h}$ at $4{ }^{\circ} \mathrm{C}$. Cells were post-fixed with $1 \%$ osmium tetroxide in $0.1 \mathrm{M}$ phosphate buffer for $1 \mathrm{~h}$. After dehydration in graded ethanol, cells were washed with propylene oxide and embedded in epoxy resin. Ultrathin sections were cut and stained with uranyl acetate and lead hydroxide, then viewed with TEM (Hitachi, H-7650). For quantitative analysis of autophagosomes, 10 random fields of each group were examined.

\section{MDC staining}

MDC was used to label autophagy vacuoles. H9c2 cells were incubated with $0.05 \mathrm{mM} \mathrm{MDC}$ at $37^{\circ} \mathrm{C}$ for $1 \mathrm{~h}$. Following incubation, cells were rinsed 3 times with PBS and analyzed immediately with a fluorescence microscopy at the excitation wavelength of $335 \mathrm{~nm}$ and the emission wavelength of $525 \mathrm{~nm}$.

\section{Western blot analysis}

Thirty $\mu$ g proteins were loaded on SDS-PAGE gels and transferred to PVDF membranes. The membranes were probed with primary antibodies overnight and then incubated with HRP-labeled secondary antibodies. Immunoreactivity was visualized using ECL (Millipore) and graphs were analyzed by the Gel-Pro Analyzer.

\section{Statistical analysis}

Data were expressed as means \pm SEM. The statistical significance was assessed by one-way ANOVA followed by Tukey's post hoc test or Student's t test using SPSS 11.5 for Windows (SPSS Inc., Chicago, IL, USA). $\mathrm{P}<0.05$ was considered statistically significant.

\section{Results}

\section{Cell viability and autophagosome formation decreased following $H R$}

H9c2 cells were subjected to $8 \mathrm{~h}$ hypoxia and reoxygenation for 4, 8, 12 and $16 \mathrm{~h}$ prior to evaluation of cell survival and autophagy. MTT assay revealed that cell viability decreased compared with control, although little difference among various reoxygenation durations (Fig. 2A). TEM is a direct qualitative identification for the observed pathologies [23]. Our data depicted that reoxygenation decreased the number of autophagosomes (Fig. 2B, C). 


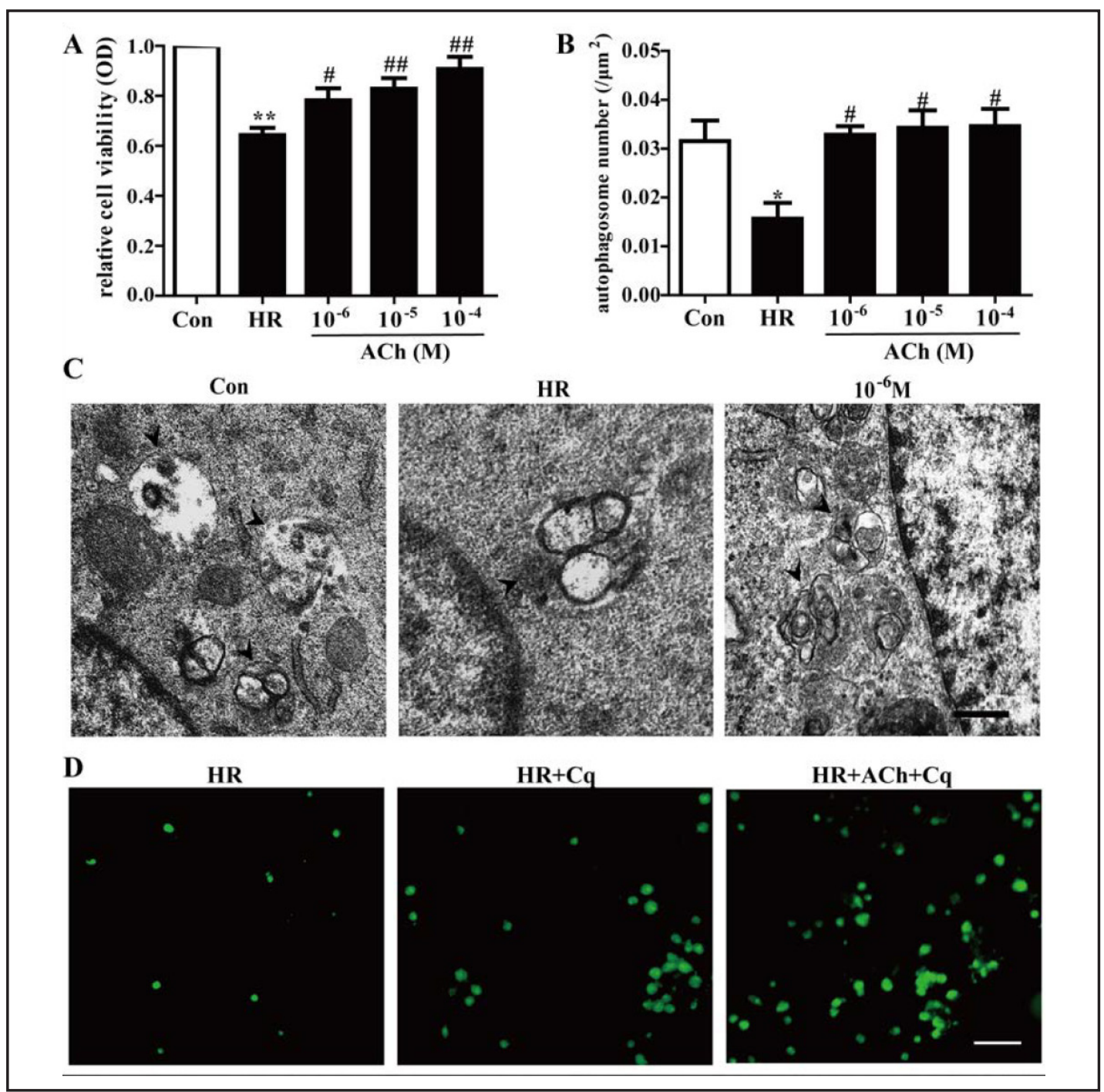

Fig. 3. Effects of ACh on cell viability and autophagosomes. (A) MTT cell viability assay. (B) Quantitative analysis of autophagosome number shown by TEM. (C) Photomicrographs showed represent samples taken from control, HR and ACh $\left(10^{-6} \mathrm{M}\right)$ groups. Arrows indicated autophagosomes. Scale bar: $0.25 \mu \mathrm{m}$. (D) Autophagic vacuoles were stained with MDC. Represent images from HR, HR+Cq, and HR+ ACh $\left(10^{-6} \mathrm{M}\right)+\mathrm{Cq}$ groups were shown. Scale bar: $25 \mu \mathrm{m}$. ${ }^{*} \mathrm{P}<0.05$ and ${ }^{* *} \mathrm{P}<0.01$ vs. control group. ${ }^{\#} \mathrm{p}<0.05$ and ${ }^{\# \#} \mathrm{p}<0.01$ vs. HR group. $n=6$ per group.

These results suggested a corroborative role of autophagy with HR. Hypoxia duration of $8 \mathrm{~h}$ followed by a reoxygenation period of $4 \mathrm{~h}$ was chosen for the remaining work.

\section{Cell viability and autophagosomes ameliorated with ACh during HR}

We went on to assess the effect of ACh on autophagy during HR. As shown in Fig. 3A, ACh $\left(10^{-6}-10^{-4} \mathrm{M}\right)$ ameliorated cell viability in a concentration dependent manner (no statistical difference between groups). TEM images revealed that H9c2 cells appeared to be normal with healthy nuclei following ACh challenge. Meanwhile, abundant autophagsosomes with double membrane and containing cytoplasmic material such as mitochondria were observed in control and ACh groups. In contrast, cells in HR group displayed few autophagosomes (Fig. $3 \mathrm{~B}, \mathrm{C}$ ). These results suggested that $\mathrm{ACh}$ was capable of activating autophagy. For the rest of the study, $10^{-6} \mathrm{M}$ ACh was chosen. Increased number of autophagosomes can be due to 


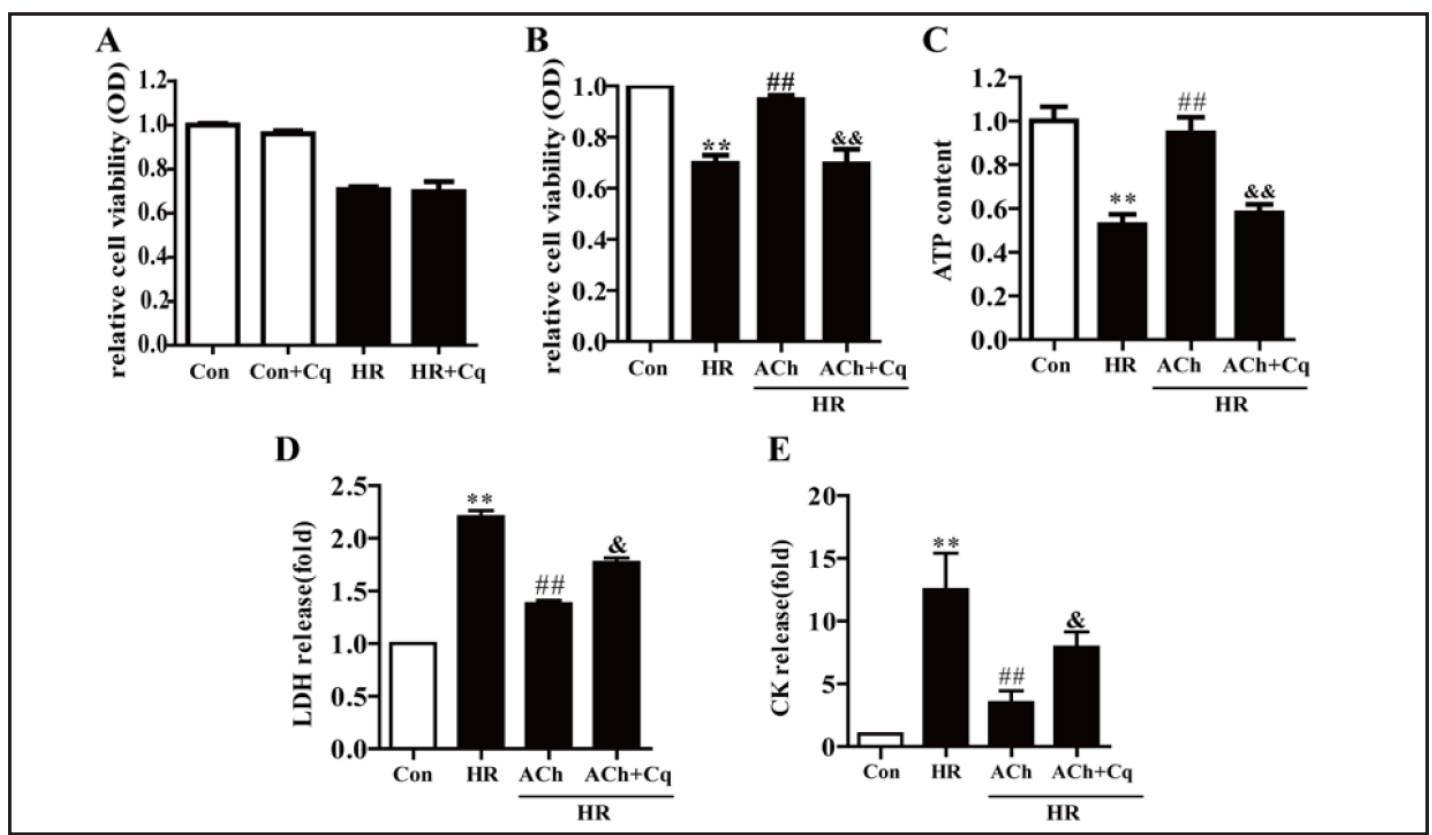

Fig. 4. Impacts of Cq on ACh-elicited cardioprotection. (A, B) Cell viability was assessed by MTT assay. (C) Intracellular ATP content with bioluminescent assay. (D, E) Cell death was measured by LDH and CK release. ${ }^{* *} \mathrm{P}<0.01$ vs. control group. ${ }^{\# \#} \mathrm{P}<0.01$ vs. HR group. ${ }^{\&} \mathrm{P}<0.05$ and ${ }^{\& \&} \mathrm{P}<0.01$ vs. ACh group. $\mathrm{n}=6$ per group.

elevated production or decreased degradation. To measure flux, we inhibited autophagosome degradation with chloroquine. As shown in Fig. 3D, chloroquine increased autophagosomes compared with HR group. ACh $\left(10^{-6} \mathrm{M}\right)$ with chloroquine further increased autophagy under $\mathrm{HR}$, indicating that ACh at least in part increased autophagy production under HR.

\section{ACh-mediated autophagy enhanced cell survival}

To confirm the potential contribution of autophagy to cardioprotection, autophagy inhibitor chloroquine was co-administered with ACh at the onset of reoxygenation. Firstly, no cytotoxic effects on $\mathrm{H} 9 \mathrm{c} 2$ cells were observed when they were treated with chloroquine either under control or HR treatment (Fig. 4A). When autophagy was induced with ACh, the cell survival by MTT assay increased than HR (Fig. 4B). HR reduced cellular ATP content when ACh largely abrogated the depletion of ATP, however, treatment with chloroquine, ATP restoration was ablated (Fig. 4C). ACh significantly reduced cell death as indicated by the reduced level of LDH and CK activity. Chloroquine did not alter the LDH and CK release compared with HR (Fig. 4D, E). Therefore, chloroquine reversed the beneficial effects of ACh against HR.

Pharmacologic inhibitors of autophagy are nonspecific and may lead to confounding results. Atg7 is essential for autophagosome formation conjugation systems [24]. To overcome these problems, Atg7 siRNA, a dominant negative inhibitor of autophagy, was carried out. Western blot analysis indicated that Atg7 siRNA treatment considerably decreased the expression of Atg7 (Fig. 5A). Atg7 siRNA per se also had no significant effect on cell injury under control or HR treatment (Fig. 5B). After the transfection efficiency was analyzed, H9c2 cells transfected with Atg7 siRNA were subjected to HR, and treated with ACh when reoxygenation. The effects of ACh on HR were attenuated by Atg7 siRNA, including that cell viability and ATP content were reduced, while cell death shown by LDH and CK release was increased compared with control siRNA (Fig. 5B, C, D and E). These data showed enhanced autophagy was important to the cardioprotection mediated by ACh. 

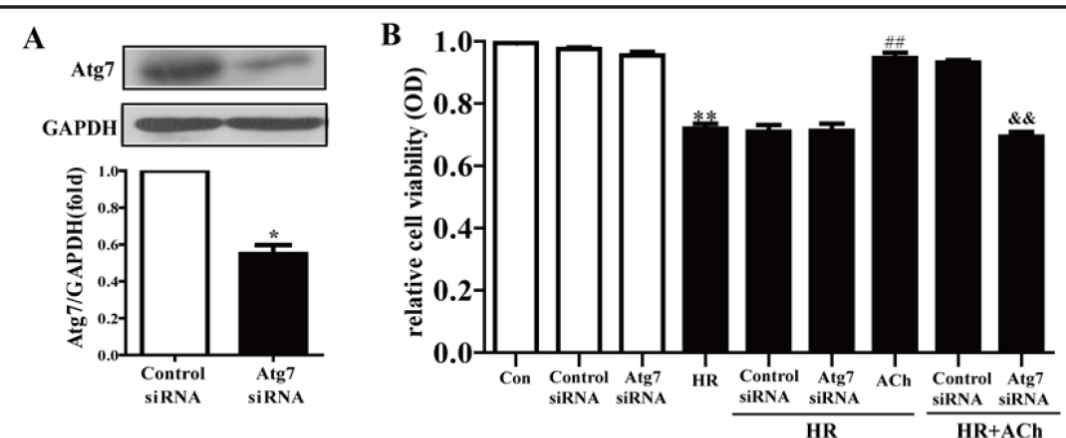

C

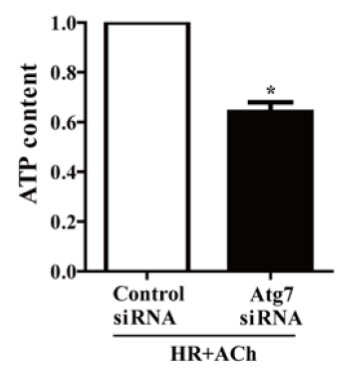

D

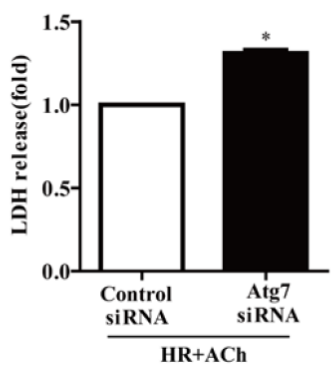

E

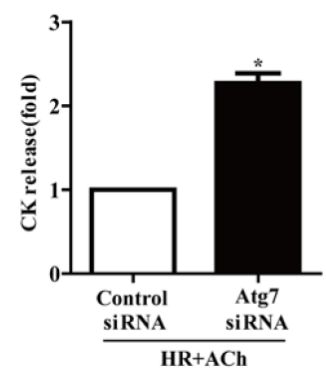

Fig. 5. Effects of Atg7 siRNA on ACh-elicited cardioprotection. (A) Western blot was performed for the detection of Atg7 knockdown. (B) Cell viability was measured by MTT assay. (C) Intracellular ATP content with bioluminescent assay. (D, E) Cell death was measured by LDH and CK release. ${ }^{*} \mathrm{P}<0.05$ vs. control siRNA group. ${ }^{* *} \mathrm{P}<0.01$ vs. control group. ${ }^{\# \#} \mathrm{P}<0.01$ vs. HR group. ${ }^{\text {\&\&}} \mathrm{P}<0.01$ vs. ACh group. $\mathrm{n}=6$ per group.

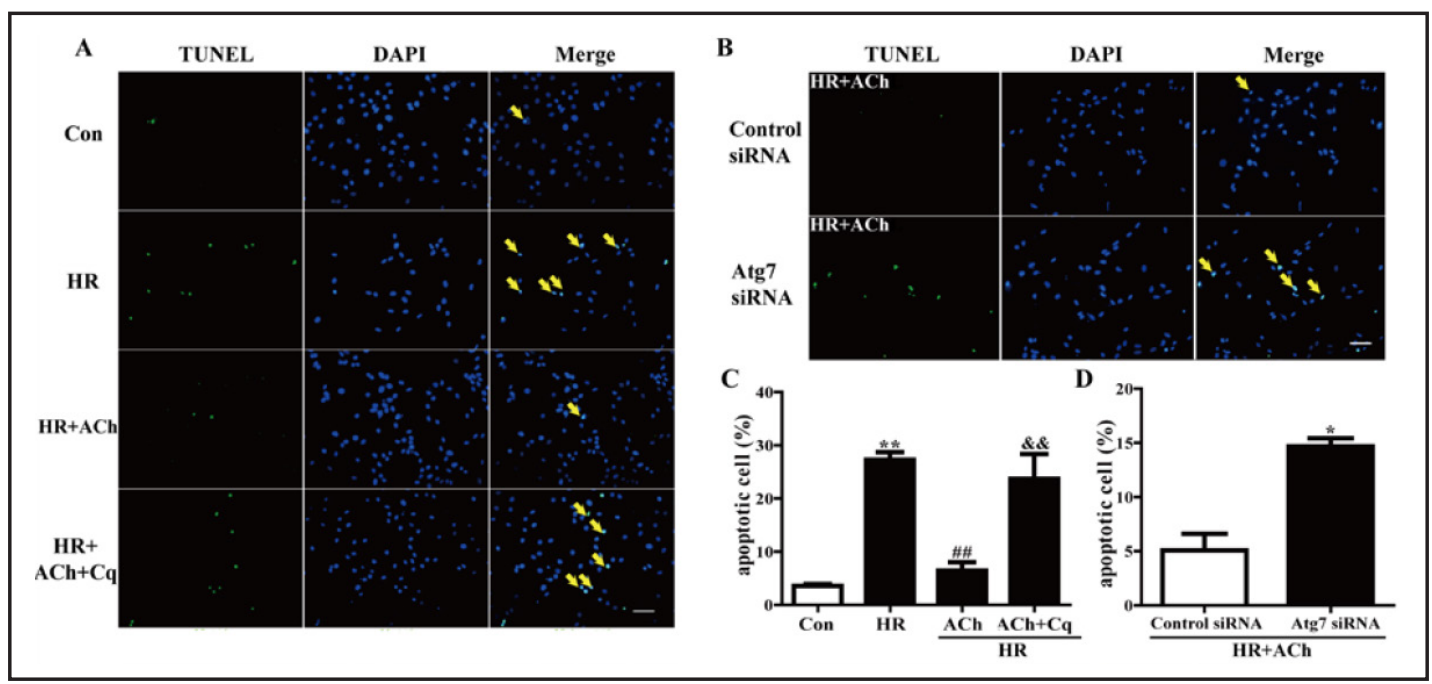

Fig. 6. Effects of ACh-induced autophagy on apoptosis with TUNEL assay. (A, B) Fluorescent microscopic images showing TUNEL and nuclear staining. Arrows denoted apoptotic cells. Scale bar: $100 \mu \mathrm{m}$. (C, D) Quantification of apoptotic cells. ${ }^{*} \mathrm{P}<0.05$ vs. control siRNA group. ${ }^{* *} \mathrm{P}<0.01$ vs. control group. ${ }^{\# \#} \mathrm{p}<0.01$ vs. HR group. ${ }^{\text {\& }} \mathrm{p}<0.01$ vs. ACh group. $\mathrm{n}=6$ per group.

\section{ACh-induced autophagy attenuated apoptosis}

To further elucidate the role of autophagy in cytoprotection, we examined apoptotic cells with TUNEL staining. When autophagy was activated with ACh, the percentage of apoptotic cells decreased. Whereas, when autophagy was inhibited as in the case of chloroquine and Atg7 siRNA, apoptosis increased (Fig. 6). These results indicated an inhibition of apoptosis brought about through an amelioration of autophagy by ACh. 


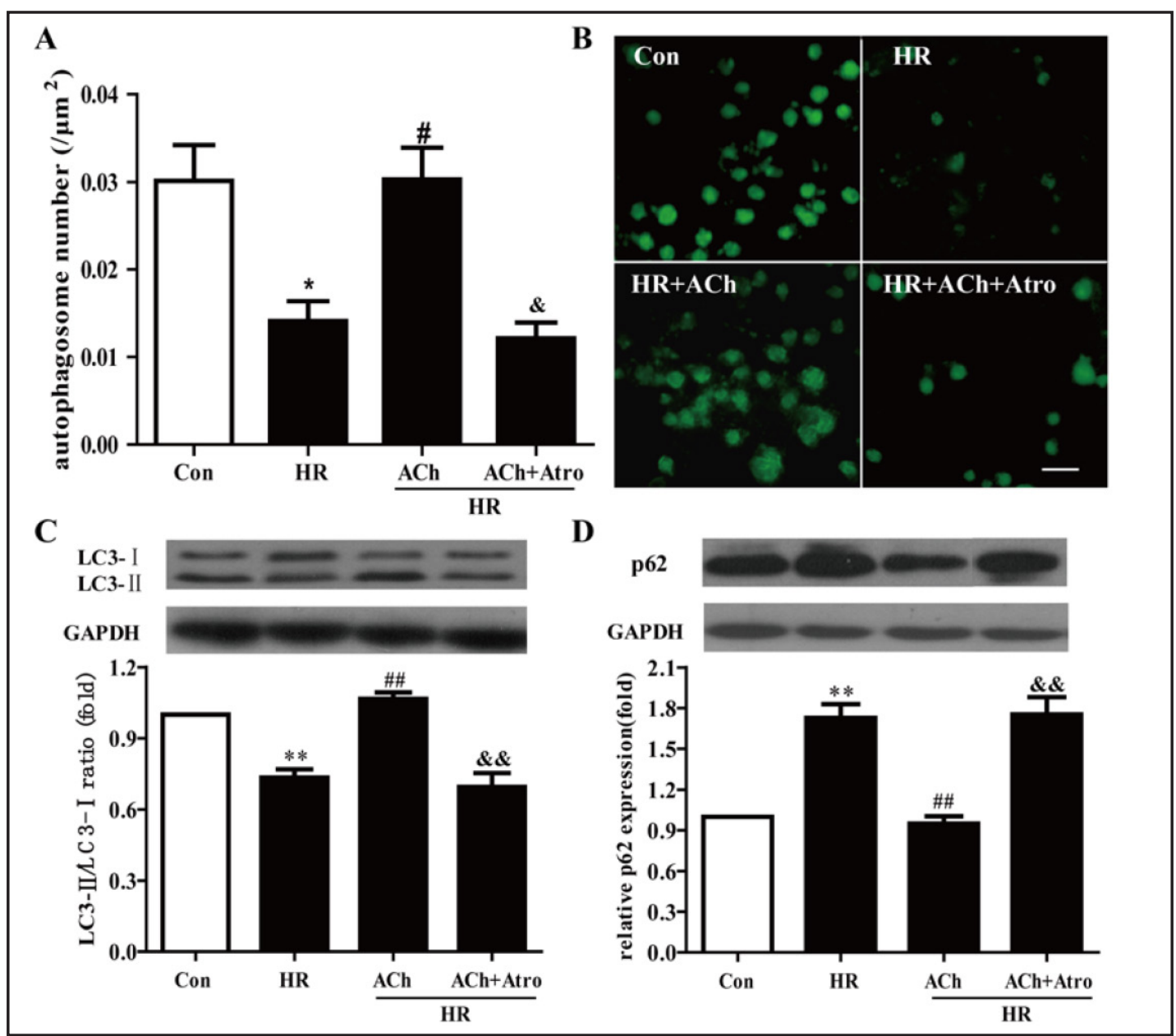

Fig. 7. Effects of muscarinic receptor on ACh-induced autophagy. (A) The number of autophagosomes shown by TEM. (B) Autophagic vacuoles were stained using MDC. Scale bar: $25 \mu \mathrm{m}$. (C, D) Western blot and quantitative evaluation of LC3 and p62 expression. ${ }^{*} \mathrm{P}<0.05$ and ${ }^{* *} \mathrm{P}<0.01$ vs. control group. ${ }^{*} \mathrm{p}<0.05$ and ${ }^{\# \#} \mathrm{p}<0.01$ vs. HR group. ${ }^{\&} \mathrm{p}<0.05$ and ${ }^{\&} \mathrm{p}<0.01$ vs. ACh group. $\mathrm{n}=6$ per group.

\section{Muscarinic receptor participated in ACh-upregulated autophagy}

The role of muscarinic receptor has been highly understood in ACh-mediated cardiac effects [25]. H9c2 cells were treated with ACh in the absence or presence of muscarinic receptor antagonist atropine under HR during reoxygenation. In line with findings in Fig. 3B and C, autophagosomes existed in ACh group, yet autophagy was mitigated by atropine (Fig. 7A). Moreover, few MDC-labeled autophagosomes were found in HR group. ACh intensified MDC fluorescence, which was abolished by atropine (Fig. 7B). LC3 is indicated as a hallmark of autophagy through the conversion of LC3-I to LC3-II. In HR group, little LC3-II was identified. ACh upregulated LC3-II, while atropine attenuated it (Fig. 7C). To evaluate autophagic flux, we monitored the levels of p62/SQSTM1, a docking protein associated with autophagic vesicles to be degraded in autophagosomes. As expected, the downregulation of p62 in ACh group was blunted by atropine (Fig. 7D). Therefore, muscarinic receptor participated in ACh-elicited autophagy.

\section{AMPK-mTOR potentiated autophagy induction with ACh}

AMPK, a major regulator of cellular energy homeostasis, is known to activate autophagy. On the other hand, inhibition of mTOR is associated with autophagy induction [26]. Our data indicated reduced AMPK phosphorylation (Thr172) and elevated mTOR phosphorylation 
Fig. 8. Effects of ACh on AMPK-mTOR signal pathway. Western blot and quantitative evaluation of (A) p-AMPK (Thr172)/ AMPK and (B) p-mTOR (S e r 24 48) / m TOR. ${ }^{*} \mathrm{P}<0.05$ vs. control group. ${ }^{\circledR} \mathrm{p}<0.05$ vs. ACh group. $n=6$ per group. group. ${ }^{\mathrm{p}}<0.05$ vs. HR

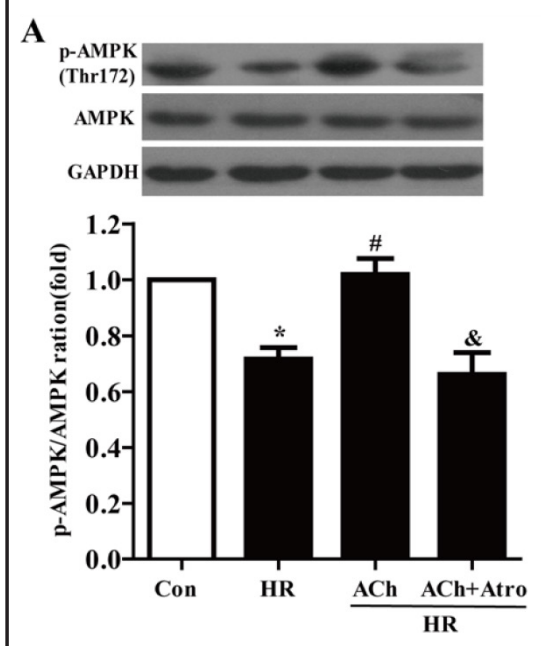

B

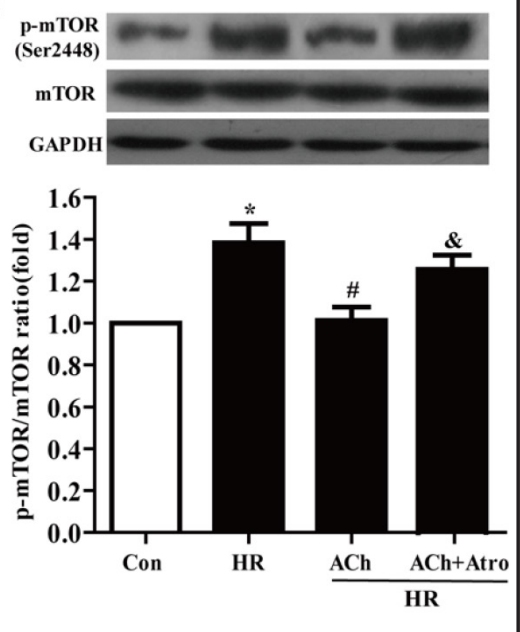

Fig. 9. Effects of AMPK siRNA on ACh-elicited autophagy and cardioprotection. (A) Western blot was performed for the detection of AMPK. (B, C) Expressions of $\mathrm{p}$-mTOR and LC3. (D) Fluorescent microscopic images showing the TUNEL staining and nuclear staining. Arrows denoted apoptotic cells. Scale bar: $100 \mu \mathrm{m}$. (E) Quantification of apoptotic cells. (F, G) LDH and $\mathrm{CK}$ release assay. ${ }^{*} \mathrm{P}<0.05$ and ${ }^{* *} \mathrm{P}<0.01$ vs. control siRNA group. $\mathrm{n}=6$ per group.

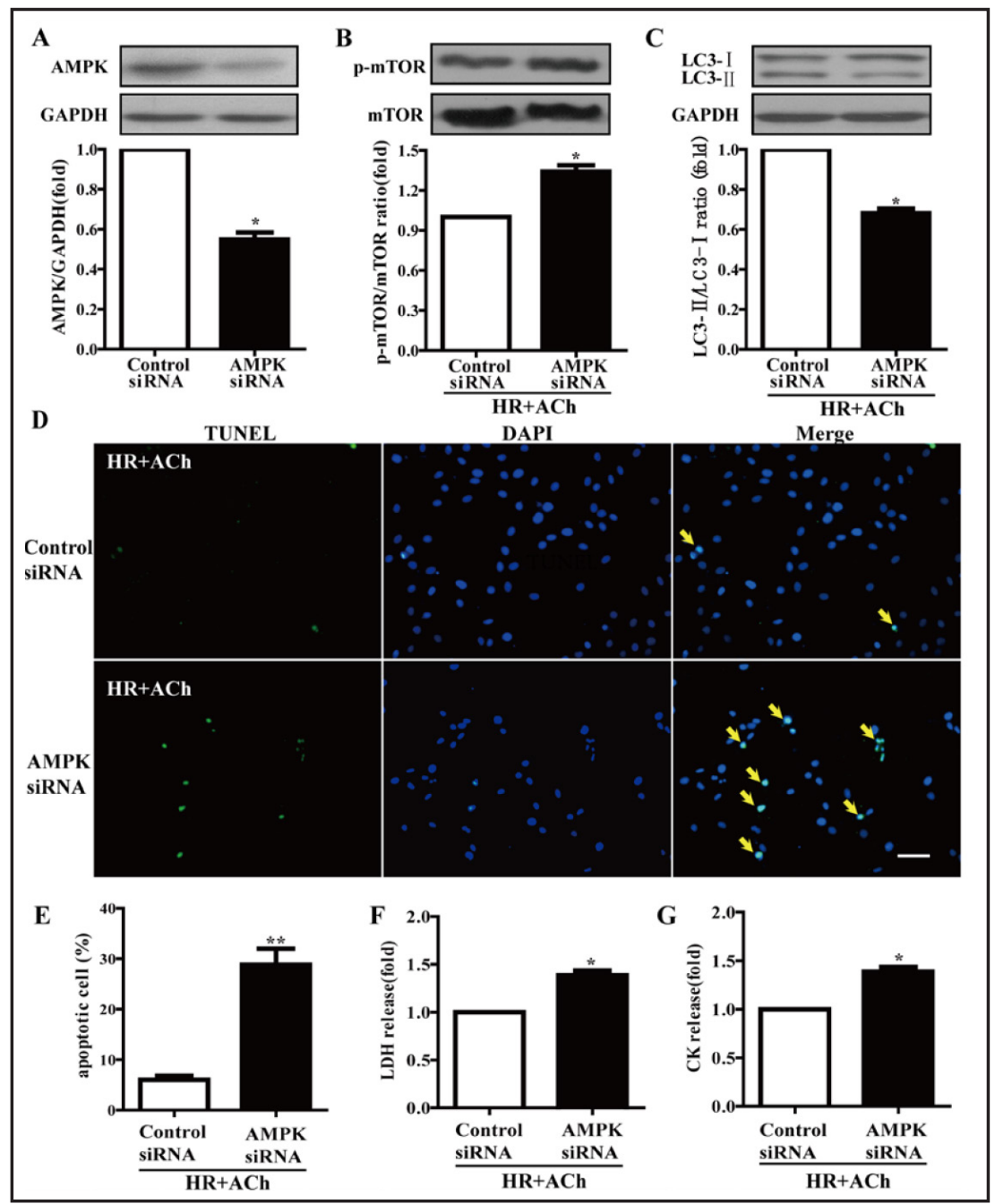

(Ser2448) in HR, while AMPK phosphorylation was significantly higher and mTOR phosphorylation was reduced prominently with ACh. Both the effects of ACh were protested by atropine (Fig. 8). These results indicated possible involvement of AMPK-mTOR signaling cascades in ACh-offered autophagy regulation under HR. 


\section{AMPK knockdown led to autophagy inhibition and cell dysfunction}

To determine if AMPK is responsible for the autophagic induction by ACh, cells were transfected with AMPK siRNA. As shown in Fig. 9A, cells exhibited a profound decrease of AMPK. No detrimental effect was found with AMPK siRNA (data not shown). Upon HR and ACh stress, knockdown of AMPK increased the ratio of p-mTOR/mTOR, while the ratio of LC3II/LC3-I was significantly reduced (Fig. 9B, C). These results demonstrated that ACh-induced autophagy was linked with AMPK pathway. AMPK-knockdown cells led to an augmentation of apoptosis, LDH and CK levels (Fig. 9D, E, F and G), corroborating the involvement of AMPK dependent autophagy in cardioprotection.

\section{Discussion}

The novel findings presented herein indicated that ACh activated cytoprotective autophagy in $\mathrm{H} 9 \mathrm{c} 2$ cells. The first intriguing phenomenon was that ACh increased the amount of autophagy observed as autophagosomes and MDC labeling vesicles. ACh also elicited autophagy function, such as increased LC3 and autophagy flux (decrease p62). The second finding was that, autophagy inhibition with chloroquine and Atg7 siRNA resulted in the loss of ACh-induced protection including improved cell survival and diminished cell death. In addition, autophagy induction was muscarinic receptor dependent, as it was blocked by atropine. Finally, AMPK siRNA increased mTOR phosphorylation and successfully inhibited autophagy, leading to increased apoptosis and cell injury. Thus it is safe to assume that AMPK-mTOR pathway participated in ACh-induced autophagy regulation (Fig. 10). These findings are consistent with the protective effects of ACh on various cardiovascular scathing conditions including HR, myocardial infarction and arrhythmia $[22,27,28]$.

H9c2 cells have been shown to retain several electrical and hormonal characteristics similar with adult cardiomyocytes. It offers an important in vitro model to analyze cardiac function including hypertrophy or heart failure [29, 30]. In current study, we employed H9c2 cells to establish HR model. The role of autophagy is still controversy in cardiovascular diseases. Autophagy as a repair mechanism is activated with low intensity stress. With increasing stress, apoptosis begins to occur. Under extreme stress, ATP depletion results in necrosis and neither autophagy nor apoptosis can progress [31]. Consistent with this, Loos et al found that the variations of autophagy are dependent on the severity and duration of ischemia and reperfusion settings [32]. Therefore a stimulation of autophagy may have been the initial response to mild stress, then was followed by decrease of autophagy as apoptosis and necrosis increased. Studies have demonstrated prominent autophagy during reperfusion in rats, while scant autophagy is also observed in pigs [33,34]. Our results indicated that 4 , $8,12,16 \mathrm{~h}$ reoxygenation led to paucity of autophagosome formation as well as declining in cell viability, suggesting that autophagy may be a possible component of cardioprotection. Javier et al found increases in Beclin1 and LC3 expressions with reduced infarct size in myocardium [34]. Yan et al. found that inhibition of autophagy exacerbated ischemia injury [35]. There seems a beneficial role of autophagy for the preservation of myocardial function during injuries.

There is ample evidence that cardiovascular disease goes along with decreased vagal tone [36]. Vagal nerve stimulation is tolerable and safe with advanced heart failure either in animals or patients [15, 37]. ACh, more than a neurotransmitter, confers protection on myocardial stresses. Autophagy, apoptosis and necrosis occur in order with intensified stresses [31]. Although vagus/ACh was proved to inhibit apoptosis and necrosis, it remained unclear whether autophagy was involved in ACh-triggered protection. Interestingly, our data provided first evidence that ACh led to activation of autophagy. Evaluation of autophagy was shown in different methods: autophagosomes from TEM and MDC fluorescence is a valid insight into what events were happening inside in a cell; western blot analysis was used to interpret the molecular changes, usually it was substantiated by the induction of LC3 and p62 degradation [38]. We confirmed that autophagy was activated by ACh morphologically and 
Fig. 10. The proposed model for the regulation of $\mathrm{ACh}$ on autophagy in a muscarinic receptor activated-AMPK-mTOR dependent manner during HR in $\mathrm{H} 9 \mathrm{c} 2$ cells.

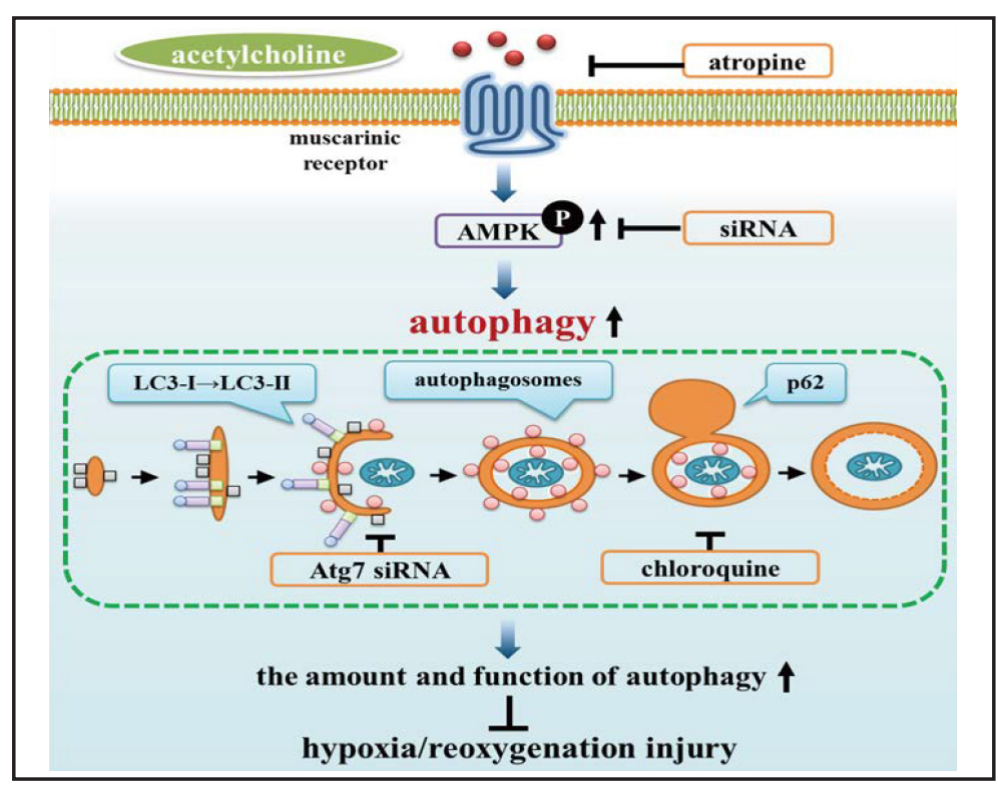

biochemically, while a low ratio of LC3-II/LC3-I and increased p62 level were observed in HR group. Notably, the muscarinic receptor antagonist atropine negated ACh-induced autophagy, which provided evidence in ACh dependent cardiac muscarinic receptor regulation.

To confirm the role of autophagy in the cardioprotection deduced by ACh, chloroquine and Atg7 siRNA were applied in HR model. Chloroquine and Atg7 siRNA potently reduced the cytoprotective effects of ACh. Our results are in agreement with previously published studies showing that chloroquine and Atg7(-/-) mice developed cardiac dysfunction by blocking autophagy activation $[39,40]$. Autophagic process can compensate for loss of energy by the regeneration of amino acids and fatty acids, which can be used for ATP synthesis [41]. ACh restored the decreased ATP content, while the effects of which were mitigated by chloroquine and Atg7 siRNA. ATP surge is associated with increased autophagy, which may suggest that increased ATP confers cytoprotection, creating a more favorable environment compared to HR group [32]. It is known that ACh can regulate apoptotic pathway, and autophagy is an apoptosis-alternative pathway, indicating the need for simultaneous detection for autophagy and apoptosis [31]. Autophagy inhibition in isolated cardiomyocytes showed increased apoptosis and necrosis, while enhanced autophagy was protective [42]. From our TUNEL assay, it is likely that a severe injury allows apoptosis to be induced, hence autophagy is depleted. These findings further supported autophagy was a homeostatic mechanism for maintaining cardiac function and provided an advantage for cellular injury.

Up-to-date, a number of cellular signaling pathways are speculated to turn on autophagy under ischemia/reperfusion [43]. Indeed, AMPK-mTOR axis is required for autophagy activation. For example, metformin and resveratrol have been found to induce autophagy by facilitating AMPK activation [12,44]. Activation of AMPK could inhibit mTOR, the negative regulator of autophagy and then stimulate autophagy. Our current study depicted overt phosphorylation of AMPK and inhibition of mTOR phosphorylation in response to ACh challenge. AMPK is a biologically participant in cardiovascular functional regulation. In addition to elevated AMPK expression with ACh during HR, AMPK knockdown with siRNA counteracted ACh-induced autophagy activation and inhibition of cellular injury, further indicated an important role of AMPK-mTOR mechanism on ACh-elicited autophagy.

In conclusion, autophagy may be essential in ACh-induced cardioprotection against ischemia/reperfusion injury. Muscarinic receptor activated-AMPK-mTOR dependent pathway provided a successful regulation mechanism for autophagy induced by ACh. Therapeutic ACh or autophagy activation may offer a promising new strategy in ischemic heart disease treatment. In addition, further studies are needed to examine the association between vagal nerve and autophagy in cardiovascular diseases. 
Zhao et al.: Acetylcholine Activates Cytoprotective Autophagy

\section{Acknowledgements}

This work is supported by the Key Project of National Natural Science Foundation of China (No. 30930105), Major International (Regional) Joint Research Project of NSFC (No. 81120108002), CMB Distinguished Professorships Award (No. F510000/G16916404) and Natural Science Foundation of Shaanxi Province (No. 2012JZ4001).

\section{References}

1 Ibanez B, Fuster V, Jimenez-Borreguero J, Badimon JJ: Lethal myocardial reperfusion injury: a necessary evil? Int J Cardiol 2010;151:3-11. Yellon DM, Hausenloy DJ: Myocardial reperfusion injury. N Engl J Med 2007;357:1121-1135. Tucka J, Bennett M, Littlewood T: Cell death and survival signalling in the cardiovascular system. Front Biosci 2012;17:248-261.

4 Kroemer G, Marino G, Levine B: Autophagy and the integrated stress response. Mol Cell 2010;40:280-293.

-5 Takemura G, Kanamori H, Goto K, Maruyama R, Tsujimoto A, Fujiwara H, Seishima M, Minatoguchi S: Autophagy maintains cardiac function in the starved adult. Autophagy 2009;5:1034-1036.

6 Hariharan N, Zhai P, Sadoshima J: Oxidative stress stimulates autophagic flux during ischemia/reperfusion. Antioxid Redox Signal 2011;14:2179-2190.

7 Levine B, Klionsky DJ: Development by self-digestion: molecular mechanisms and biological functions of autophagy. Dev Cell 2004;6:463-477.

8 Petrovski G, Das S, Juhasz B, Kertesz A, Tosaki A, Das DK: Cardioprotection by endoplasmic reticulum stress-induced autophagy. Antioxid Redox Signal 2011;14:2191-2200.

- Jiang M, Liu K, Luo J, Dong Z: Autophagy is a renoprotective mechanism during in vitro hypoxia and in vivo ischemia-reperfusion injury. Am J Pathol 2010;176:1181-1192.

10 Pattison JS, Osinska H, Robbins J: Atg7 induces basal autophagy and rescues autophagic deficiency in CryABR120G cardiomyocytes. Circ Res 2011;109:151-160.

11 Yitzhaki S, Huang C, Liu W, Lee Y, Gustafsson AB, Mentzer RM Jr, Gottlieb RA: Autophagy is required for preconditioning by the adenosine A1 receptor-selective agonist CCPA. Basic Res Cardiol 2009;104:157167.

12 Gurusamy N, Lekli I, Mukherjee S, Ray D, Ahsan MK, Gherghiceanu M, Popescu LM, Das DK: Cardioprotection by resveratrol: a novel mechanism via autophagy involving the mTORC2 pathway. Cardiovasc Res 2010;86:103-112.

13 Sroka K: On the genesis of myocardial ischemia. Z Kardiol 2004;93:768-783.

14 Cisternas JR, Valenti VE, Alves TB, Ferreira C, Petenusso M, Breda JR, Pires AC, Tassi N, de Abreu LC: Cardiac baroreflex is already blunted in eight weeks old spontaneously hypertensive rats. Int Arch Med 2010;3:2.

-15 Schwartz PJ, De Ferrari GM, Sanzo A, Landolina M, Rordorf R, Raineri C, Campana C, Revera M, AjmoneMarsan N, Tavazzi L, Odero A: Long term vagal stimulation in patients with advanced heart failure: first experience in man. Eur J Heart Fail 2008;10:884-891.

16 Zhang Y, Kakinuma Y, Ando M, Katare RG, Yamasaki F, Sugiura T, Sato T: Acetylcholine inhibits the hypoxiainduced reduction of connexin43 protein in rat cardiomyocytes. J Pharmacol Sci 2006;101:214-222.

17 Kakinuma Y, Ando M, Kuwabara M, Katare RG, Okudela K, Kobayashi M, Sato T: Acetylcholine from vagal stimulation protects cardiomyocytes against ischemia and hypoxia involving additive non-hypoxic induction of HIF-1alpha. Febs Lett 2005;579:2111-2118.

18 Kawada T, Yamazaki T, Akiyama T, Inagaki M, Shishido T, Zheng C, Yanagiya Y, Sugimachi M, Sunagawa K: Vagosympathetic interactions in ischemia-induced myocardial norepinephrine and acetylcholine release. Am J Physiol Heart Circ Physiol 2001;280:H216-H221.

19 Uemura K, Li M, Tsutsumi T, Yamazaki T, Kawada T, Kamiya A, Inagaki M, Sunagawa K, Sugimachi M: Efferent vagal nerve stimulation induces tissue inhibitor of metalloproteinase-1 in myocardial ischemiareperfusion injury in rabbit. Am J Physiol Heart Circ Physiol 2007;293:H2254-H2261.

20 Lu J, Zang WJ, Yu XJ, Jia B, Chorvatova A, Sun L: Effects of postconditioning of adenosine and acetylcholine on the ischemic isolated rat ventricular myocytes. Eur J Pharmacol 2006;549:133-139. 


\section{Cellular Physiology Cell Physiol Biochem 2013;32:601-613

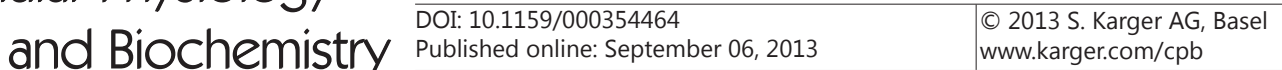 \\ Zhao et al.: Acetylcholine Activates Cytoprotective Autophagy}

21 Liu JJ, Li DL, Zhou J, Sun L, Zhao M, Kong SS, Wang YH, Yu XJ, Zhou J, Zang WJ: Acetylcholine prevents angiotensin II-induced oxidative stress and apoptosis in H9c2 cells. Apoptosis 2011;16:94-103.

22 Miao Y, Zhou J, Zhao M, Liu JJ, Sun L, Yu XJ, He X, Pan XY, Zang WJ: Acetylcholine attenuates hypoxia/ reoxygenation-induced mitochondrial and cytosolic ROS formation in H9c2 cells via M2 acetylcholine receptor. Cell Physiol Biochem 2013;31:189-198.

23 Swanlund JM, Kregel KC, Oberley TD: Investigating autophagy: quantitative morphometric analysis using electron microscopy. Autophagy 2010;6:270-277.

-24 Burman C, Ktistakis NT: Autophagosome formation in mammalian cells. Semin Immunopathol 2010;32:397-413.

-25 Harvey RD: Muscarinic receptor agonists and antagonists: effects on cardiovascular function. Handb Exp Pharmacol 2012;208:299-316.

26 Xie Z, He C, Zou MH: AMP-activated protein kinase modulates cardiac autophagy in diabetic cardiomyopathy. Autophagy 2011;7:1254-1255.

27 Ando M, Katare RG, Kakinuma Y, Zhang D, Yamasaki F, Muramoto K, Sato T: Efferent vagal nerve stimulation protects heart against ischemia-induced arrhythmias by preserving connexin 43 protein. Circulation 2005;112:164-170.

28 Tsutsumi T, Ide T, Yamato M, Kudou W, Andou M, Hirooka Y, Utsumi H, Tsutsui H, Sunagawa K: Modulation of the myocardial redox state by vagal nerve stimulation after experimental myocardial infarction. Cardiovasc Res 2008;77:713-721.

29 Zordoky BN, El-Kadi AO: H9c2 cell line is a valuable in vitro model to study the drug metabolizing enzymes in the heart. J Pharmacol Toxicol Methods 2007;56:317-322.

30 Watkins SJ, Borthwick GM, Arthur HM: The H9c2 cell line and primary neonatal cardiomyocyte cells show similar hypertrophic responses in vitro. In Vitro Cell Dev Biol Anim 2011;47:125-131.

-31 Nishida K, Yamaguchi 0, Otsu K: Crosstalk between autophagy and apoptosis in heart disease. Circ Res 2008;103:343-351.

32 Loos B, Genade S, Ellis B, Lochner A, Engelbrecht AM: At the core of survival: autophagy delays the onset of both apoptotic and necrotic cell death in a model of ischemic cell injury. Exp Cell Res 2011;317:1437-1453.

-33 Sadoshima J: The role of autophagy during ischemia/reperfusion. Autophagy 2008;4:402-403.

- 34 Sala-Mercado JA, Wider J, Undyala VV, Jahania S, Yoo W, Mentzer RJ, Gottlieb RA, Przyklenk K: Profound cardioprotection with chloramphenicol succinate in the swine model of myocardial ischemia-reperfusion injury. Circulation 2010;122:S179-S184.

-35 Yan W, Zhang H, Bai X, Lu Y, Dong H, Xiong L: Autophagy activation is involved in neuroprotection induced by hyperbaric oxygen preconditioning against focal cerebral ischemia in rats. Brain Res 2011;1402:109121.

-36 Bibevski S, Dunlap ME: Evidence for impaired vagus nerve activity in heart failure. Heart Fail Rev 2011;16:129-135.

-37 Li M, Zheng C, Sato T, Kawada T, Sugimachi M, Sunagawa K: Vagal nerve stimulation markedly improves long-term survival after chronic heart failure in rats. Circulation 2004;109:120-124.

-38 Mizushima N: Methods for monitoring autophagy. Int J Biochem Cell Biol 2004;36:2491-2502.

-39 Mizushima N, Yoshimori T, Levine B: Methods in mammalian autophagy research. Cell 2010;140:313-326.

40 Takagi H, Matsui Y, Sadoshima J: The role of autophagy in mediating cell survival and death during ischemia and reperfusion in the heart. Antioxid Redox Signal 2007;9:1373-1381.

41 Sciarretta S, Hariharan N, Monden Y, Zablocki D, Sadoshima J: Is autophagy in response to ischemia and reperfusion protective or detrimental for the heart? Pediatr Cardiol 2011;32:275-281.

-42 Hamacher-Brady A, Brady NR, Gottlieb RA: Enhancing macroautophagy protects against ischemia/ reperfusion injury in cardiac myocytes. J Biol Chem 2006;281:29776-29787.

43 Papandreou I, Lim AL, Laderoute K, Denko NC: Hypoxia signals autophagy in tumor cells via AMPK activity, independent of HIF-1, BNIP3, and BNIP3L. Cell Death Differ 2008;15:1572-1581.

44 Shi WY, Xiao D, Wang L, Dong LH, Yan ZX, Shen ZX, Chen SJ, Chen Y, Zhao WL: Therapeutic metformin/ AMPK activation blocked lymphoma cell growth via inhibition of mTOR pathway and induction of autophagy. Cell Death Dis 2012;3:e275. 